EDUR • Educação em Revista. 2020; 36:e223402

DOI: http://dx.doi.org/10.1590/0102-223402

() (1) https://creativecommons.org/licenses/by/4.0/

ORCID: https://orcid.org/0000-0002-1460-8282

ADRIANA MOREIRA DA ROCHA VEIGA ${ }^{2}$

ORCID: https://orcid.org/0000-0002-5804-3375

MARCELO PEDROSO DA ROZA ${ }^{3}$

ORCID: https://orcid.org/0000-0002-2771-4893

ARTIGO

\title{
BLENDED LEARNING: REVISÃO SISTEMÁTICA DA LITERATURA EM PERIÓDICOS CIENTÍFICOS INTERNACIONAIS (2015 - 2018)
}

\begin{abstract}
RESUMO: O Blended Learning, ao combinar as aprendizagens presencial e on-line, é uma oportunidade de os professores integrarem as tecnologias digitais, no meio acadêmico, de modo ponderado, sem romper com as estratégias e práticas que já utilizam, mas buscando incorporá-las a esses modos contemporâneos de aprender. Com o objetivo de descobrir o movimento atual das pesquisas, o contexto educacional em que se aplicam e os assuntos abordados, este estudo traz uma revisão sistemática da literatura sobre Blended Learning, elaborada a partir das publicações entre 2015 e 2018, em periódicos científicos de visibilidade internacional. As publicações foram organizadas em tópicos com base em suas temáticas, servindo, também, para exemplificar os achados e as questões de pesquisa. Como resultado, este estudo evidencia um cenário crescente de experimentações com Blended Learning, realizadas, geralmente, a partir de iniciativas de professores, e onde predominam as experiências voltadas ao design instrucional e aos resultados dos alunos no Ensino Superior.
\end{abstract}

Palavras-chave: Ensino híbrido, Métodos de ensino e aprendizagem, Revisão sistemática da literatura.

\section{BLENDED LEARNING: A SYSTEMATIC LITERATURE REVIEW IN INTERNATIONAL SCIENTIFIC JOURNALS (2015 - 2018)}

\begin{abstract}
Blended Learning, by combining the face-to-face and on-line learning, is an opportunity for teachers to integrate digital technologies, in the educational environment, in a thoughtful way without disrupting with strategies and practices they already use but seeking to incorporate them into these contemporary ways of learning. With the objective of discovering current research, including the educational context in which they are applied along with the subjects addressed, this study provides a systematic review of the literature on blended learning, developed from the publications from 2015 to 2018 , in scientific journals of international visibility. The publications identified were organized into topics based on themes of interest and that exemplify the findings and research questions. As a result,

\footnotetext{
${ }^{1}$ Instituto Federal Farroupilha, IFFAR. Alegrete, RS, Brasil.<jiani.roza@iffarroupilha.edu.br>

${ }^{2}$ Universidade Federal de Santa Maria, UFSM. Santa Maria, RS, Brasil. <adrianaufsm@gmail.com>

${ }^{3}$ Instituto Federal Farroupilha, IFFAR. Alegrete, RS, Brasil. <mroza@iffarroupilha.edu.br> Educação em Revista|Belo Horizonte|v.36|e223402|2020
} 
this dissertation demonstrated an increasing scenario of international experimentation with blended learning, generally developed through a variety of diverse teacher initiatives, and where the experiments focused on instructional design and student predominates.

Keywords: Blended learning, Teaching and learning methods, Systematic literature review.

\section{BLENDED LEARNING: REVISIÓN SISTEMÁTICA DE LA LITERATURA EN PERIÓDICOS CIENTÍFICOS INTERNACIONALES (2015 - 2018)}

RESÚMEN: El Blended Learning, al combinar los aprendizajes presencial y on line, es una oportunidad para que los profesores integren las tecnologías digitales, en el medio académico, de modo ponderado, sin romper con las estrategias y prácticas que utilizan ya, pero buscando incorporarlas a esos modos contemporáneos de aprender. Con el objetivo de descobrir el movimiento actual de las investigaciones, el contexto educacional en el que se aplican y los asuntos abordados, este estudio presenta una revisión sistemática de la literatura sobre Blended Learning, elaborada a partir de las publicaciones entre 2015 y 2018 , en periódicos científicos de visibilidad internacional. Se han organizado las publicaciones en tópicos basados en sus temáticas, lo que ayudó además a ejemplificar los hallazgos y las cuestiones de investigación. Como resultado, este estudio evidencia un escenario creciente de experimentaciones con Blended Learning, realizadas, generalmente, a partir de iniciativas de profesores, y donde predominan las experiencias direccionadas al design instruccional y a los resultados de los alumnos en la Enseñanza Superior.

Palabras clave: Blended learning, Métodos de enseñanza y aprendizaje, Revisión sistemática de la literatura.

\section{INTRODUÇÃO}

O Blended Learning (BL), assim como sua própria natureza, é um conceito híbrido. As TIC, ao evoluírem, vão reconfigurando as possibilidades educacionais, devido à riqueza de interações, de recursos e de experiências que elas (as TIC) nos permitem explorar. Assim, torna-se inevitável que se estudem e se planejem formas de integrá-las aos processos de ensino e aprendizagem.

$\mathrm{E}$, nessa esfera, o $B L$, uma mescla entre ensinos presencial e on-line, desponta como um caminho viável, pois não rompe com as estratégias de ensino conhecidas e fortemente adotadas, mas as expande e provoca a integração das tecnologias digitais na educação, em convergência com uma prática pedagógica que resulte no desenvolvimento dos aprendizes.

Embora não haja uma definição única e universal a respeito de $B L$, a sua concepção tem evoluído com o tempo, com as tecnologias, com as necessidades atuais e com as compreensões dos pesquisadores. Segundo Graham (2006), o BL deve refletir a ideia de combinação de dois modelos de ensino-aprendizagem historicamente separados: o tradicional - com sistemas de aprendizagem F2F (da expressão em inglês face to face - e o modelo a distância - com os sistemas de aprendizagem distribuídos - Ambientes Virtuais de Aprendizagem (AVA), plataformas adaptativas, gamificação. Ou seja, é da intersecção dos dois modelos que o BL emerge.

Garrison e Vaughan (2008) definem BL como a integração orgânica de abordagens e tecnologias on-line, cuidadosamente selecionadas e complementares. A expressão "orgânica" deve ser entendida como fundamentado na prática, enquanto que a expressão "cuidadosamente" suscita um repensar significativo da forma como devemos abordar as experiências de aprendizagem. Esse conceito de BL enfatiza o papel autotransformador do professor.

Para Horn, Staker e Christensen (2015), o BL é uma abordagem formal e inovadora para a educação, que busca: potencializar a aprendizagem por meio da tecnologia; equilibrar a aprendizagem individual com a coletiva; integrar os espaços físicos com os virtuais; atribuir um papel mais interativo ao professor e mais autônomo aos estudantes; personalizar a aprendizagem, ao permitir que atenda ao ritmo e ao estilo de aprendizado dos estudantes, com flexibilidade de tempo e espaço. Essa definição dá 
ênfase à personalização e à utilização de metodologias ativas. Nesse sentido, a definição de $B L$ evoluiu para abarcar um conjunto mais rico de estratégias e dimensões de aprendizagem (BACICH; NETO; TREVISANI, 2015).

Portanto, a concepção de BL se baseia em uma ideia de educação híbrida - mista -, que mescla múltiplas estratégias, práticas, espaços, indivíduos, tecnologias e culturas, de maneira a potencializar a construção do conhecimento. Em relação a isso, Garrison e Vaughan (2013) observaram que a definição de $B L$ deve ser mais inclusiva do que restritiva, porém é importante adotar uma definição que permita flexibilidade pedagógica aos professores.

Desse modo, entendemos o BL como a confluência entre modelos, metodologias e práticas pedagógicas da educação presencial e da educação on-line combinadas e potencializadas pelas TIC, preferencialmente de modo simultâneo. Segundo Moran (2015), a educação sempre foi blended, sempre combinou vários tempos, atividades, metodologias, públicos. Esse processo está sendo mais percebido, ampliado e aprofundado com as TIC. Dessa forma, a "mistura" mais complexa é integrar o que vale a pena aprender, para quê e como fazer.

Nesse entendimento, o BL tem sido visto como o "novo modelo normal" (NORBERG; DZIUBAN; MOSKAL, 2011), com um número crescente de estudos sendo compartilhado sobre o tema. Assim, nas duas últimas décadas, a produção científica relacionada a $B L$ tem sido frequente em periódicos ligados à Educação e à Tecnologia Educacional.

Dessa forma, ao considerarmos as revisões de literatura sobre o assunto, encontramos os trabalhos de Halverson et al. (2012) que investigaram, para o período de 2000 a 2011, os (10) livros, os (25) capítulos de livros editados e os (50) artigos mais citados sobre $B L$, bem como os periódicos nos quais esses estudos foram publicados (suas descobertas ajudaram a determinar onde as conversas sobre $B L$ estavam acontecendo e quais pesquisadores estavam na vanguarda dessas conversas, destacando, ainda, um aumento na implementação institucional do BL em contextos de Educação Superior).

Na sequência, Drysdale et al. (2013) examinaram (205) teses e dissertações escritas, até 2011, nos EUA e Canadá, disponíveis na base de dados ProQuest, no domínio do BL, para as quais identificaram tendências teóricas, metodológicas e demográficas envolvendo a temática, enfatizando, ainda, lacunas e oportunidades de pesquisas. Além disso, os autores agruparam as questões de pesquisa abordadas nos estudos em categorias (tópicos), as quais chamaram de tendências temáticas. Nessa pesquisa, mais da metade dos estudos abordou os resultados do ensino-aprendizagem realizado com $B L$ e se evidenciou uma área aberta para pesquisas na Educação Básica e a necessidade de contribuições mais teóricas, exclusivas dos contextos de BL.

Posteriormente, Halverson et al. (2014), usando codificação aberta e análise temática, investigaram o conteúdo dos (50) artigos e dos (25) capítulos de livros de maior impacto sobre BL para o período de 2002 a 2011, a fim de determinar as metodologias, as questões de pesquisa e os referenciais teóricos abordados nesses trabalhos, discutindo, também, as implicações desses resultados para pesquisa em BL. A investigação apresenta os dez tópicos principais de pesquisa em $B L$, com base na análise temática construída, e sugere uma falta de coesão teórica entre as pesquisas para os trabalhos investigados. Esse estudo foi complementar ao desenvolvido por Halverson et al. (2012), com ênfase no que as conversas sobre $B L$ realmente tratam.

Desse modo, neste artigo, apresentamos uma Revisão Sistemática da Literatura (RSL), que busca refletir acerca do movimento da produção científica internacional sobre $B L$, especificamente em artigos de periódicos internacionais, revisados por pares, para o período de 2015 a 2018, a fim de incentivar a integração das TIC nos espaços educacionais contemporâneos, a partir da perspectiva do $B L$.

Nesse sentido, procuramos responder as seguintes questões: Em que contextos (Educação Básica, Educação Superior, Desenvolvimento Profissional) estão ocorrendo as pesquisas em BL? Quais temas são mais frequentes nos estudos com $B L$ ? Quais questões de pesquisa relacionadas a $B L$ estão sendo abordadas? Quais periódicos internacionalmente reconhecidos são fontes de pesquisa atuais para a temática? 
Entendemos que a justificativa para uma RSL se dá pelo seu potencial em apresentar direções já sinalizadas pela comunidade científica com relação a $B L$, bem como em revelar lacunas e possibilidades de investigação futuras para os pesquisadores.

\section{METODOLOGIA}

Foi realizada uma RSL seguindo as orientações propostas em Depaepe, Verschaffel e Kelchtermans (2013) e em Costa e Zoltowski (2014). O levantamento foi conduzido no Portal de Periódicos da Coordenação de Aperfeiçoamento de Pessoal de Nível Superior (CAPES), que disponibiliza às instituições de ensino e pesquisa do Brasil, através do acesso remoto via comunidade acadêmica federada, os artigos completos de revistas de impacto científico internacional.

O recorte temporal desta investigação compreende o período de 2015 a 2018, em razão das discussões em torno do tema serem recentes no campo da educação no Brasil e, assim, proporcionarem um overview das pesquisas no âmbito internacional para o período investigado.

Dessa forma, o processo de construção da revisão foi constituído pelas seguintes etapas: delimitação das questões a serem pesquisadas; escolha da fonte de dados; definição da palavra-chave para a busca; busca e armazenamento dos resultados; seleção de artigos com base nos critérios de inclusão e exclusão; extração dos dados dos artigos selecionados; avaliação dos artigos; síntese e interpretação dos dados (COSTA; ZOLTOWSKI, 2014). As etapas foram desenvolvidas por dois pesquisadores, de modo a minimizar os vieses na interpretação.

Tendo em vista que a terminologia original, em inglês, é usada com frequência nas literaturas nacional e internacional e expressa nos resumos em inglês, realizamos a busca utilizando como palavrachave "Blended Learning". Desse modo, considerando as questões de pesquisa propostas na revisão, a busca foi configurada iniciando por: "buscar assunto"; na sequência, selecionamos a opção "busca avançada", onde foi construída a seguinte expressão: "no título (é exato) 'Blended Learning' AND no assunto contém 'education'". Essa escolha foi tomada de modo a viabilizar que o BL esteja contemplado predominantemente no conteúdo do artigo e cujo foco seja educacional.

No "tipo de material" foi selecionado "artigos" e, em "data de publicação", foi informado "01/01/2015", como data inicial, e "31/12/2018”, como data final. Em "idioma", foi deixado "qualquer idioma". Nesse momento da busca, não se limitou a consulta a uma base de dados específica dentro do Portal de Periódicos da CAPES. A busca foi conduzida em fevereiro de 2019.

Essa busca resultou em 411 artigos, para os quais foi possível selecionar a opção "mostrar somente os periódicos revisados por pares", restando assim 340 artigos, distribuídos em 173 periódicos diferentes. A partir dessa seleção, outros critérios de exclusão foram aplicados com base na leitura dos títulos e dos resumos e, quando necessário, do texto completo, mas de maneira exploratória:

a) artigos derivados da mesma pesquisa, publicados em anos diferentes;

b) artigos repetidos;

c) artigos que abordam $B L$, porém com aplicação fora da área educacional;

d) relatórios técnicos ou projetos na forma de resumo;

e) artigos cujo resumo não estivesse disponível em inglês, português ou espanhol;

Dessa etapa, resultaram 297 artigos, os quais foram importados para o software NVivo 12 (QSRINTERNATIONAL, 2019), que auxiliou na organização, codificação e análise do corpus constituído. Para auxiliar na categorização e análise dos artigos, recorremos à Análise de Conteúdo na perspectiva de Bardin (2010). E, para apoiar o gerenciamento das referências, foi utilizado o software EndNote X9.

\section{Critérios de extração e análise de dados}

Para identificarmos em que contextos se situam as pesquisas, agrupamos os 297 artigos em categorias definidas a priori, exclusivas e autoexcludentes, a saber: Educação Básica, Educação Superior, Desenvolvimento Profissional e Outros. A fim de realizar a categorização nessas unidades, observamos o título do periódico, o título da publicação, as palavras-chave, e procedemos à leitura dos resumos. 
Quando não explicitado nesses elementos, o texto foi lido na íntegra. Dois pesquisadores realizaram a categorização de modo independente. Incertezas e ajustes foram resolvidos por meio de negociação.

Nos artigos classificados na categoria "Educação Básica", comumente designada por K-12 nos EUA, Canadá e Austrália, estão as pesquisas referentes ao público do Ensino Fundamental e Médio. Já, na categoria da "Educação Superior", reunimos as pesquisas voltadas ao público dos cursos de graduação e pós-graduação (estudantes, professores, tutores).

Na categoria "Desenvolvimento Profissional", foram associadas as propostas e iniciativas de formação (continuada e em serviço) de professores e de outros profissionais (médicos, enfermeiros, odontólogos) envolvendo ensino-aprendizagem por meio do BL. Embora boa parte das iniciativas de desenvolvimento profissional seja proveniente da Educação Superior, agrupamos à parte as pesquisas relacionadas a esse contexto, como forma de explicitar o uso de BL para promover o desenvolvimento profissional. E, por fim, a categoria "Outros" reúne os artigos científicos que não se enquadravam nas categorias criadas; dentre eles, os artigos de revisão e conceituação teórica sobre BL.

Para responder quais periódicos, internacionalmente reconhecidos, com foco na Educação e na Tecnologia Educacional, são fontes de pesquisa atuais para a temática, classificamos inicialmente a relação dos periódicos onde foram publicados os 297 artigos. Dos 297 artigos, primeiramente foram extraídos os nomes dos periódicos nos quais eles foram publicados. Nessa relação, foram identificados 124 títulos de periódicos diferentes. Considerando o alto número de revistas que continham uma ou duas publicações no máximo (94 títulos), utilizamos como critério de seleção a frequência de publicações relacionadas a $B L$, selecionando aquelas que publicaram três ou mais artigos, em anos diferentes, excluindo-se, assim, revistas que apresentassem dossiês ou edições especiais.

Por fim, consultamos o QUALIS ${ }^{4}$ do periódico na área da Educação e/ou Ensino e o Journal Citation Reports (JCR), visando identificar o fator de impacto relevante nessas áreas. Revistas para as quais não foi possível localizar o JCR ou o QUALIS foram, então, descartadas. Desse modo, restaram dez periódicos, os quais denominamos Top Ten, apresentados nos resultados.

Com relação a quais tópicos são mais frequentes nas pesquisas, tomamos como base as tendências tópicas para as questões de pesquisa no domínio de BL propostas por Drysdale et al. (2013) e complementadas por Halverson et al. (2014), a saber: design instrucional, resultado dos alunos, disposição, tecnologia, desenvolvimento profissional, comparação, demografia, interação, outros. Cada uma contendo vários subtópicos agrupados por similaridade e abrangência. Essa categorização foi estruturada a partir da análise temática e da codificação aberta.

Assim, apoiados pela Análise de Conteúdo, realizamos a leitura na íntegra, e fragmentos dos textos foram utilizados na codificação. Para isso, foi criada inicialmente a estrutura de árvore de nós, no software NVivo (Figura 1), para codificar os artigos e chegarmos à frequência dos tópicos nas publicações em $B L$ e às questões de pesquisa.

\footnotetext{
${ }^{4}$ Qualis é o método de classificação utilizado pela CAPES para estratificação da qualidade da produção intelectual dos programas de pós-graduação. A base de dados do Journal Citation Reports (JCR) é uma fonte de pesquisa reconhecida por avaliar periódicos indexados na $W$ eb of Science. Por meio da JCR, é possível verificar os periódicos mais citados em uma determinada área e a relevância da publicação para a comunidade científica, através do Fator de Impacto (FI). 
Figura 1 - Estrutura criada no NVivo para codificar as publicações

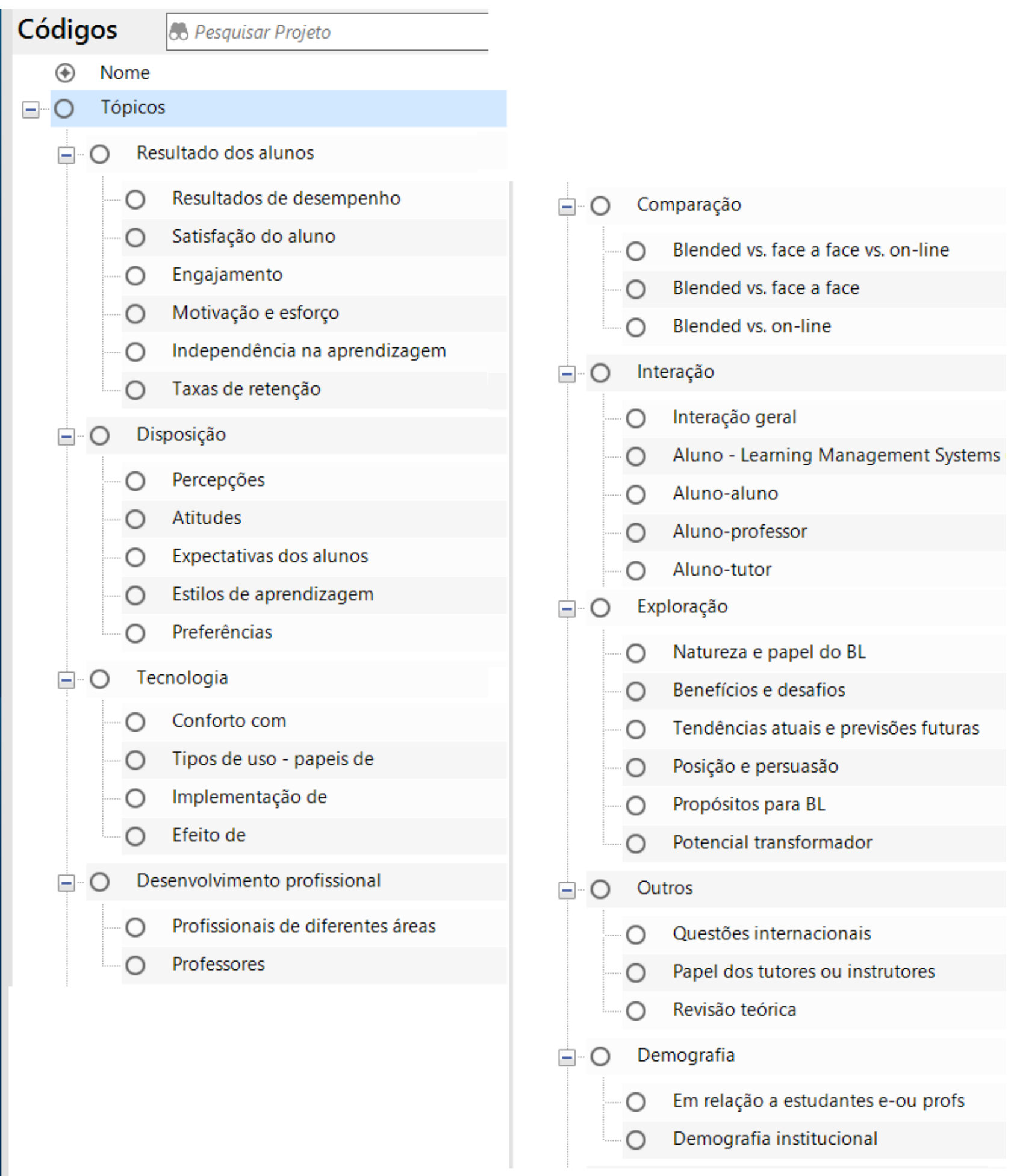

Fonte: Os autores (2019).

\section{RESULTADOS E DISCUSSÕES}

Começamos a análise verificando a distribuição dos artigos por ano de publicação, uma vez que o BL tem sido visto como tendência para a educação. O Gráfico 1 traz esse resultado. 
Gráfico 1 - $\mathrm{N}^{\circ}$ de artigos publicados por ano com a expressão Blended Learning no título

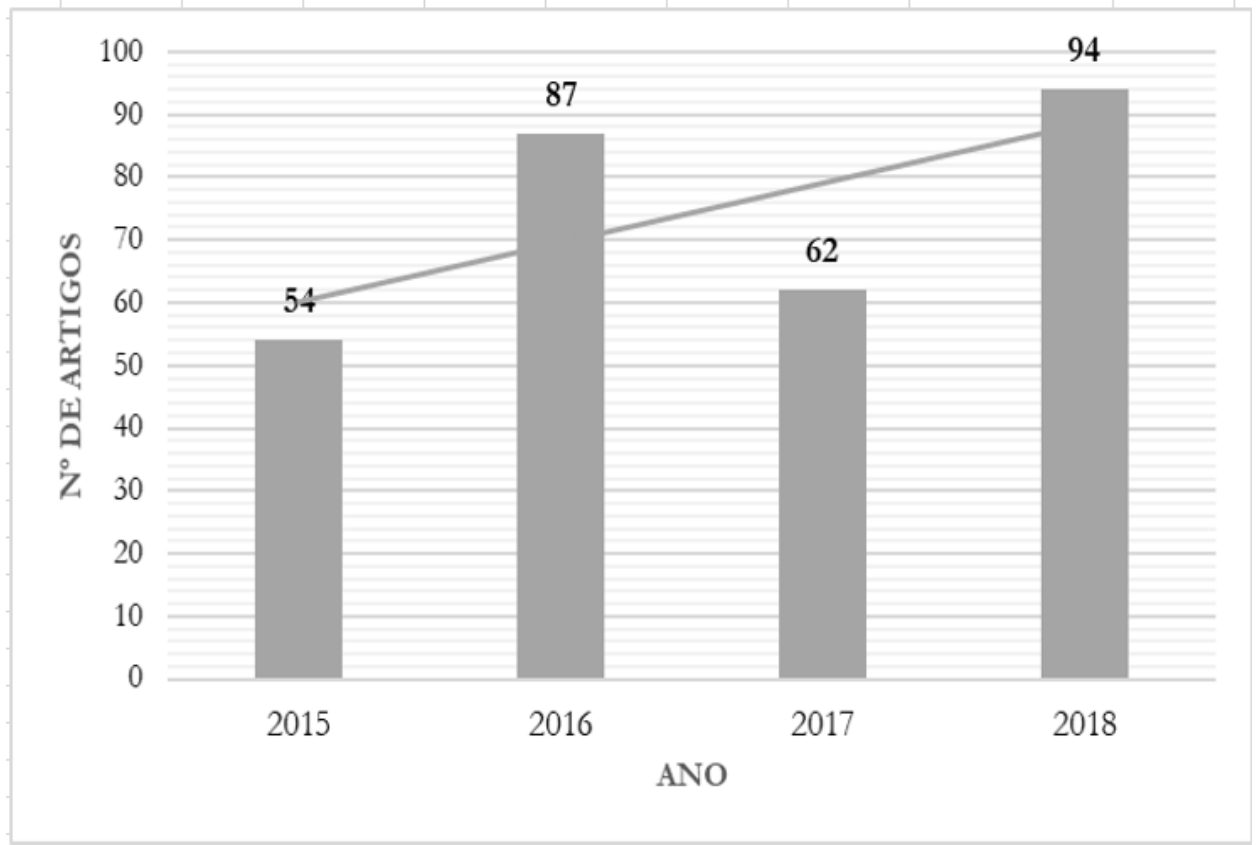

Fonte: Os autores (2019).

Considerando que a busca envolveu a expressão "Blended Learning", presente no título dos artigos, podemos perceber um volume expressivo de publicações em torno desse tema, com maior destaque para os anos de 2016 e 2018, que podem servir de fonte de pesquisa para o assunto.

$\mathrm{Na}$ sequência, realizamos a categorização dos artigos com relação ao contexto educacional de aplicação das pesquisas. Desse modo, o Gráfico 2 traz a representação dessa classificação, organizada conforme ano de publicação do artigo.

\section{Gráfico 2 - $\mathrm{N}^{\circ}$ de artigos científicos com Blended Learning, por contexto educacional}

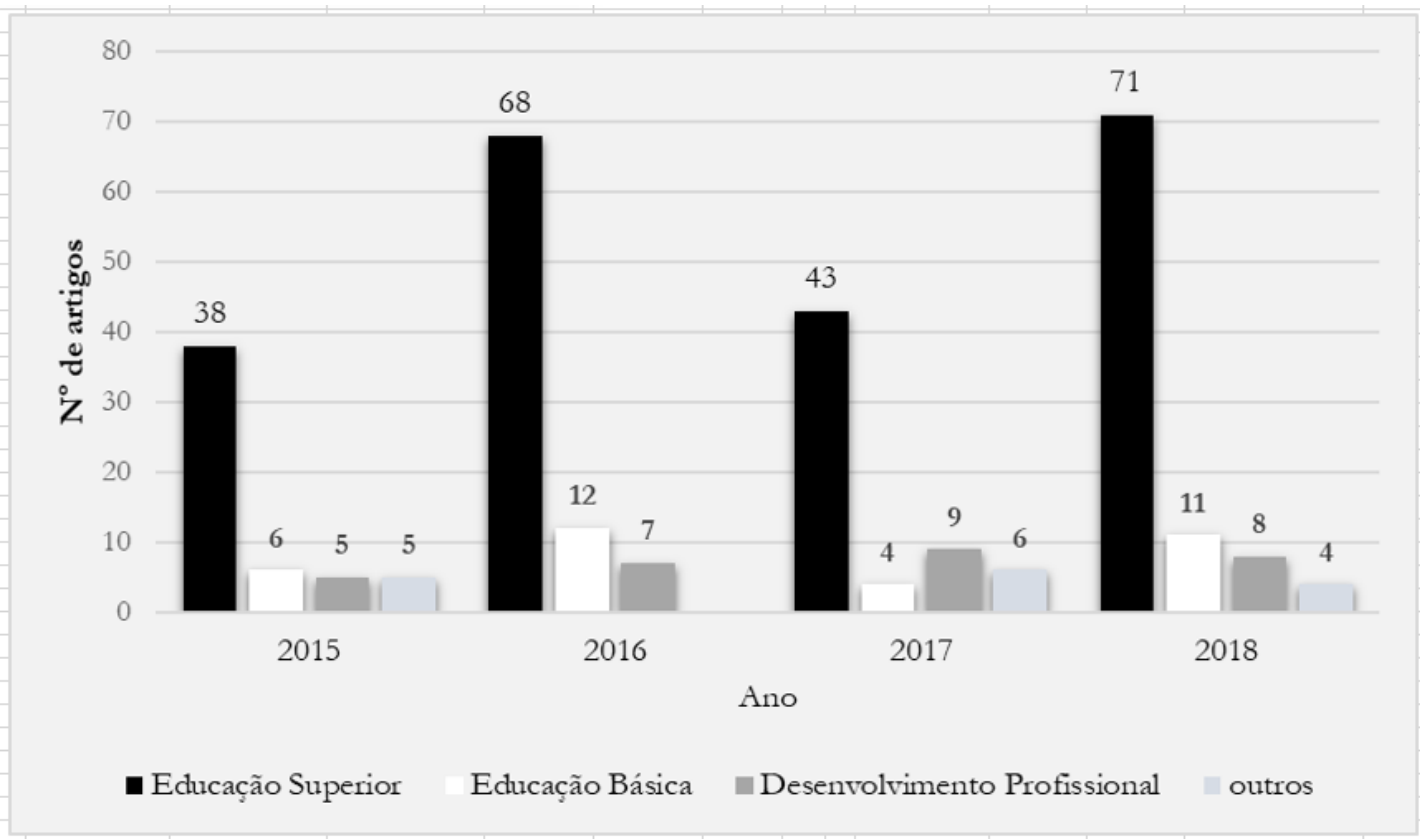

Fonte: Os autores (2019). 
Podemos observar, no Gráfico 2, que as publicações científicas, no âmbito da Educação Superior, continuam sendo o foco da maioria das pesquisas, correspondendo, em nossa busca, a 220 artigos, ou seja, 74,07\% das publicações. Nas descobertas de Halverson et al. (2012), a Educação Superior já constituía o contexto das publicações mais citadas sobre $B L$, com $66,1 \%$ das produções analisadas, focadas exclusivamente nesse nível de ensino.

Conforme Halverson et al. (2012), que investigaram os estudos mais mencionados na primeira década de $B L$, há uma lacuna significativa de investigações voltadas à Educação Básica, visto que, na época, somente $1,8 \%$ das produções foram identificadas no contexto de Educação Básica. Quando organizada por ano, a pesquisa em contextos K-12 não foi consistentemente presente até 2008 (DRYSDALE et al., 2013).

Embora o acréscimo no número de artigos voltados à Educação Básica seja notório, o estudo do BL relacionado a esse nível educacional é muito pouco explorado ou divulgado nos periódicos de visibilidade internacional. Em nosso estudo, esse gap pode ser observado, pois encontramos 33 artigos voltados à Educação Básica, correspondendo a 11,11\% das publicações analisadas e, nessas, quase a totalidade direcionada ao Ensino Médio.

As pesquisas relacionadas a iniciativas ou programas de formação inicial e/ou continuada, categorizadas em "Desenvolvimento Profissional", mostram-se com um movimento discreto, porém contínuo, ao longo dos quatro anos, com 29 artigos (9,77\%) identificados nessa categoria. Por outro lado, boa parte desses trabalhos utilizaram o BL como meio para promover a formação (capacitação) dos professores em suas áreas de atuação ou interesse. Desse modo, identificamos uma lacuna em pesquisas que investiguem modelos de formação docente para o uso de $B L$.

Por fim, na categoria "Outros", estão agrupados 15 artigos (correspondendo a 5,05\% do total) que não se encaixaram nas categorias anteriormente citadas ou cujo estudo não tenha explicitado o nível educacional, podendo, assim, servir a qualquer categoria anterior, tais como alguns estudos de revisão.

Embora esta análise seja resultante exclusivamente de artigos científicos compreendidos entre 2015 e 2018, ela corrobora os achados de pesquisa anteriormente aludidos e evidencia que, nas pesquisas voltadas à Educação Básica e à formação dos docentes envolvendo BL, há um amplo espaço a ser explorado e divulgado.

Cabe ressaltar, ainda, que autores frequentemente citados na literatura referente a $B L$, como Garrison e Kanuka (2004), Graham (2006), Garrison e Vaughan (2008), MacDonald (2008), entre outros, apresentam diversas pesquisas direcionadas ao Ensino Superior. Contudo, Christensen, Horn e Staker (2013) e Horn e Staker (2015), embora tenham estudos voltados à Educação Básica, mais precisamente a K-12, também são citados em estudos com foco na Educação Superior. Dessa forma, podemos supor que a literatura disponível contribui para o crescimento do BL em um determinado nível.

\section{Periódicos internacionais que constituem fontes de pesquisa para Blended Learning}

Apresentamos, no Quadro 1, uma relação dos Top Ten (os dez mais) dos periódicos que, no período de 2015 a 2018, mais publicaram sobre o BL.

Nessa seleção apresentada, das revistas citadas em Halverson et al. (2012), que mais publicaram sobre o assunto, se mantêm em destaque quatro delas, a saber: Computers \& Education, British Journal of Educational Technology, The Internet and Higher Education. No geral, são periódicos que promovem a disseminação do conhecimento em ensino e aprendizagem, em tecnologias emergentes e em diferentes contextos de e-Learning.

Observamos, também, que, dos periódicos científicos listados no Quadro 1, quatro deles podem ser encontrados nos estratos A1 ( $\left.\mathrm{n}^{\circ}: 1,3,4\right)$ e B2 (n: 6) do QUALIS Periódicos, na área de avaliação da Educação e/ou Ensino. Além disso, todos os dez periódicos possuem Fator de Impacto reconhecido pelo JCR/ISI e são indexados em importantes bases de dados, como Scopus, Web of Science, Educational Resource Information Center (ERIC), ProQuest, Scientific Electronic Library Online (SciElo), Directory Open Access Journals (DOAJ), British Education Index, PSYCINFO e ScienceDirect. Isso nos revela que há 
publicação científica qualificada relacionada a $B L$ em nível mundial, o que contribui para a compreensão e disseminação das experiências.

Quadro 1 - Top Ten dos periódicos com maior $\mathrm{n}^{\circ}$. de publicações com Blended Learning

\begin{tabular}{|c|l|c|c|}
\hline Posição & Nome do periódico & ISSN & N. $^{\text {o de artigos }}$ \\
\hline $1^{\mathrm{a}}$ & BMC Medical Education & $1472-6920$ & 11 \\
\hline $2^{\mathrm{a}}$ & Education and Information Technologies & $1360-2357$ & 9 \\
\hline $3^{\mathrm{a}}$ & Computers \& Education & $0360-1315$ & 7 \\
\hline $4^{\mathrm{a}}$ & Advances in Physiology Education & $1043-4046$ & 6 \\
\hline $5^{\mathrm{a}}$ & Electronic Journal of e-Learning (EJEL) & $1479-4403$ & 6 \\
\hline $6^{\mathrm{a}}$ & Online Learning Journal & $1939-5256$ & 5 \\
\hline $7^{\mathrm{a}}$ & The Internet and Higher Education & $1096-7516$ & 4 \\
\hline $8^{\mathrm{a}}$ & Internet Journal of Educational Technology in Higher Education & $2365-9440$ & 4 \\
\hline $9^{\mathrm{a}}$ & British Journal of Education Technology & $1467-8535$ & 3 \\
\hline $10^{\mathrm{a}}$ & Australasian Journal of Educational Technology & $1449-5554$ & 3 \\
\hline
\end{tabular}

Fonte: Os autores (2019).

Por outro lado, é perceptível que a publicação atual em torno do BL está disseminando-se em múltiplas direções, tanto em termos de periódicos que abrigam as pesquisas quanto em termos de país de origem das mesmas, se consideramos os 124 títulos de periódicos que abrigam os artigos.

Nesse sentido, com relação aos países nos quais as pesquisas têm origem, pela leitura dos artigos, foi possível identificar que há estudos sobre BL nos diferentes continentes. Na América, encontramos pesquisas de instituições de ensino: nos EUA, no Canadá, no Brasil, na Colômbia, no México; na Europa, os artigos são oriundos de estudos na: Alemanha, Inglaterra, Dinamarca, Irlanda, Ucrânia; na Ásia, os estudos vêm: da Índia, da China, do Nepal, da Tailândia, da Malásia, da Turquia, da Rússia, da Indonésia; na Oceania, temos pesquisas na Croácia e na Austrália; e, na África, as contribuições vêm da Tanzânia e da Uganda. Porém, é importante enfatizar que os periódicos americanos, ingleses, australianos e canadenses concentraram boa parte das publicações em torno da temática advindas de inúmeras instituições de ensino, presentes nos diferentes continentes.

Embora muitos desses estudos representem casos isolados de experiências com $B L$, eles sinalizam que esse(a) modelo/estratégia pedagógico(a) está expandindo-se e tornando-se cada vez mais presente e necessário(a) em diferentes sistemas educacionais e contextos sociais.

Ainda em relação aos periódicos, identificamos um volume expressivo de publicações em periódicos científicos e acadêmicos, ligadas às áreas da Educação: Médica, Farmacêutica, em Enfermagem, em Odontologia, que relatam experiências de ensino e aprendizagem com BL. Cabe destacar que, embora não tenha sido relacionada no Top Ten, devido aos critérios aplicados, a Revista Iberoamericana de Educación a Distancia (RIED) reuniu, na edição de janeiro de 2018, volume 21, um dossiê temático sobre Blended Learning, apresentando 11 artigos.

\section{Tópicos frequentes nas pesquisas com Blended Learning}

Para codificarmos as publicações de acordo com as categorias (tópicos) apresentadas por Drysdale et al. (2013) e Halverson et al. (2014), e para uma melhor análise do cenário das pesquisas relacionadas a $B L$, realizamos a leitura dos 297 artigos, fazendo o cruzamento entre título, palavras-chave, objetivo da pesquisa e conclusões. Nesse momento, extraímos, ainda, as questões e/ou objetivos de pesquisa dos artigos, a fim de determinar a sua classificação dentro de um determinado tópico, com base na Análise de Conteúdo. 
Dessa forma, publicações que abordaram mais de um tópico foram codificadas para o tópico dominante, levando-se em consideração as questões de pesquisa e/ou declaração de propósitos. Dois pesquisadores categorizaram independentemente cada publicação. Nessa etapa, dúvidas e desacordos foram solucionados com a ajuda de um terceiro pesquisador, quando necessário. Com isso, no Quadro 2, são apresentados os números e porcentagens de artigos compilados para cada tópico (categoria).

Quadro 2 - Número e frequência dos tópicos nas publicações em Blended Learning

\begin{tabular}{|c|c|c|c|}
\hline Tópicos & Subtópicos & $\mathbf{N}^{\circ}$ de artigos & Frequência \\
\hline $\begin{array}{l}\text { Design } \\
\text { Instrucional }\end{array}$ & $\begin{array}{l}\text { Modelos, estratégias e melhores práticas, processo de } \\
\text { design, implementação e ambiente e estrutura do curso. }\end{array}$ & 85 & $28,6 \%$ \\
\hline $\begin{array}{l}\text { Resultado dos } \\
\text { Alunos }\end{array}$ & $\begin{array}{l}\text { Resultados de desempenho, satisfação do aluno, } \\
\text { engajamento, motivação e esforço, independência na } \\
\text { aprendizagem e taxas de retenção. }\end{array}$ & 52 & $17,5 \%$ \\
\hline Disposição & $\begin{array}{l}\text { Percepções, atitudes, preferências, expectativas dos } \\
\text { alunos e estilos de aprendizagem. }\end{array}$ & 37 & $12,5 \%$ \\
\hline Tecnologia & $\begin{array}{l}\text { Conforto com, efeito de, tipos de usos / papéis de, e } \\
\text { implementação de. }\end{array}$ & 34 & $11,4 \%$ \\
\hline $\begin{array}{l}\text { Desenvolvimento } \\
\text { Profissional }\end{array}$ & Profissionais de diferentes áreas, professores. & 22 & $7,4 \%$ \\
\hline Comparação & $\begin{array}{l}\text { Blended vs. face a face vs. on-line, blended vs. face a face e } \\
\text { blended vs. on-line. }\end{array}$ & 20 & $6,7 \%$ \\
\hline Exploração & $\begin{array}{l}\text { Natureza e papel do } B L \text {, benefícios e desafios, } \\
\text { tendências atuais e previsões futuras, posição/persuasão, } \\
\text { propósitos para } B L \text { e/ou potencial transformador. }\end{array}$ & 19 & $6,4 \%$ \\
\hline Demografia & $\begin{array}{l}\text { Em relação a estudantes e/ou professores, demografia } \\
\text { institucional. }\end{array}$ & 10 & $3,4 \%$ \\
\hline Interação & $\begin{array}{l}\text { Interação geral, aluno-Learning Management Systems (LMS), } \\
\text { aluno-aluno, aluno-professor, aluno-tutor, colaboração, } \\
\text { comunidade e presença social. }\end{array}$ & 8 & $2,7 \%$ \\
\hline Outros & $\begin{array}{l}\text { Questões internacionais, papel dos tutores (ou } \\
\text { instrutores), revisão teórica. }\end{array}$ & 10 & $3,4 \%$ \\
\hline
\end{tabular}

Fonte: Elaborado, pelos autores, a partir de Drysdale et al. (2013) e Halverson et al. (2014).

O Quadro 2 nos mostra que o tópico "Design Instrucional", que abrange o estudo de modelos, estratégias e melhores práticas para utilização do BL, bem como o estudo dos processos de design, implementação e organização estrutural de disciplinas (ou cursos) híbridos, continua sendo o tópico mais presente nas publicações em $B L$, correspondendo a $28,6 \%$ dos artigos analisados. Desde Halverson et al. (2014), esse tópico é o mais frequente nas publicações envolvendo BL, tendo atingido $41,2 \%$ das publicações. De modo geral, podemos dizer que os trabalhos atuais estão mais voltados a relatos de experiência com $B L$ inserido em modelos predominantemente presenciais.

O tópico "Resultado dos Alunos", que considerou o desempenho, a satisfação, a motivação, o esforço e a independência da aprendizagem em cursos/disciplinas blended, esteve presente em 17,5\% das questões de pesquisa, atingindo o segundo tópico mais explorado. O desempenho dos estudantes em cursos/disciplinas híbridas, em comparação aos exclusivamente presenciais, é, de longe, o tema mais investigado nesse conjunto. Em Halverson et al. (2014), 28,2\% das publicações mais citadas apontavam para esse tópico, ocupando o $4^{\circ}$ lugar na relação dos tópicos mais citados em questões de pesquisa com $B L$.

Em nosso trabalho, o tópico "Disposição" atingiu 12,5\% dos artigos analisados. De modo geral, as pesquisas voltadas a identificar as preferências, atitudes e expectativas dos alunos em relação à tecnologia nas aulas demonstram o interesse em manter os momentos de interação face a face (professorestudante e estudante-estudante), sem abdicar das oportunidades que a tecnologia promove. Além disso, o estudo das percepções dos alunos é foco da maioria dos trabalhos.

Embora ocupe o $2^{\circ}$ lugar no ranking dos tópicos, na pesquisa apresentada por Halverson et al. (2014), com 31,8\%, a "Disposição" continua oferecendo um bom número de trabalhos desenvolvidos quando o assunto é BL. Contudo, a lacuna relacionada às disposições dos professores pode ser novamente observada, uma vez que, desde Halverson et al. (2014), esse gap nas pesquisas já era 
identificado. Ainda nesse tópico, conhecer os estilos de aprendizagem dos alunos e as possibilidades de personalização que o $B L$ pode proporcionar também é um campo de pesquisa em aberto.

Nos artigos analisados, as questões de pesquisa voltadas às "Tecnologias" envolvidas na prática do $B L$ alcançaram 11,4\%. No geral, esses trabalhos compreenderam o estudo e análise da aplicação de diferentes recursos computacionais (Modular Object Oriented Distance Learning (MOODLE), Massive Open Online Courses (MOOC), Mobile Learning) para dar suporte às atividades e à comunicação online.

Os tópicos "Desenvolvimento Profissional", "Comparação" e "Exploração" receberam atenção de 7,4\%, 6,7\% e 6,4\% das publicações, respectivamente (conforme Quadro 2). Comparando ao trabalho apresentado por Halverson et al. (2014), "Tecnologia" e "Comparação" atingiram 17,6\% (5 e $6^{\circ}$ lugares, respectivamente) cada um, enquanto que o "Desenvolvimento Profissional" alcançou apenas 3,5\% ( $9^{\circ}$ lugar) dos estudos; por outro lado, o tópico "Exploração" chegou a 29,4\% dos artigos e capítulos de livros pesquisados pelo autor ( $3^{\circ}$ tópico em sua lista). Se considerarmos que a pesquisa de Halverson et al. (2014) abrangia a primeira década de estudos, é compreensível que houvesse um maior interesse em conceituar, teorizar e estruturar o que significava o $B L$, considerando as inovações tecnológicas e pedagógicas que eles propunham.

Atualmente, o desenvolvimento profissional realizado em um formato híbrido apresenta-se como uma alternativa viável de atualização on-line (seja em termos geográficos ou econômicos) a uma gama diversificada de profissionais, com maior incidência, em nossa pesquisa, sobre aqueles ligados à área da Saúde ou Educação, sendo o $5^{\circ}$ tópico mais explorado em nossa análise.

Porém, no que se refere ao "Desenvolvimento Profissional", conforme estudo desenvolvido por Roza (2019), há uma carência de estudos cujo foco seja a formação docente para a integração do BL, seja em contextos de formação inicial ou continuada.

Porter et al. (2016) aplicaram a estrutura de adoção institucional de BL apresentada por Graham, Woodfield e Harrison (2013), bem como a difusão de inovações de Rogers (2003), para determinar em que grau a estratégia institucional, a estrutura e o suporte influenciavam a disposição dos professores da Universidade Brigham Young Idaho (BYU-I) em adotar o BL e se a categoria de adoção de inovação dos professores do Ensino Superior afeta as medidas que facilitam ou impedem a adoção de $B L$.

No que diz respeito à "Demografia", o número de pesquisas que envolvem aspectos demográficos relacionados aos estudantes, professores/tutores se mantém escasso: apenas 3,4\% em nosso estudo. Do mesmo modo, o tópico "Outros" - que inclui: questões internacionais (dentre elas, a inserção do $B L$ para estudantes estrangeiros); o papel dos tutores em cursos blended; ou, ainda, revisões teóricas sobre $B L$ - obteve essa porcentagem.

Em nossa pesquisa, o tópico "Interação" foi identificado em apenas 2,7\% dos artigos. Embora, no estudo de Halverson et al. (2014), esse tópico tenha sido apresentado em 14,1\% das questões de pesquisa, aspectos ligados à interatividade em espaços blended, seja em relação ao conteúdo (e suas múltiplas formas de apresentação/exploração) seja entre os alunos ou entre estudantes e professor/tutor, dentro e fora da instituição, a interação é uma questão coadjuvante em boa parte das pesquisas.

Cabe ressaltar que, na pesquisa de Halverson et al. (2014), as publicações que abordaram mais de um tópico foram codificadas em vários tópicos, de acordo com sua análise. Ou seja, as porcentagens apresentadas pelo autor ultrapassaram 100\%. Porém, optamos por categorizar cada artigo em um tópico apenas, considerando as questões de pesquisa e/ou objetivos do estudo, o título, as palavras-chave, a fim de compormos a nossa análise. Dessa forma, a posição ocupada pelos tópicos serve de orientação para identificarmos os caminhos já percorridos, os novos, e os territórios a serem desbravados quando o assunto envolve $B L$.

A seguir, buscamos sintetizar alguns artigos capazes de exemplificar cada tópico. Desse modo, o Quadro 3 reúne uma amostra estratificada, destacando as questões de pesquisa presentes em alguns dos artigos analisados. Nessa etapa, os exemplos foram extraídos de publicações com texto completo em inglês, espanhol ou português, relacionadas à Educação Superior, devido ao alto volume de produções direcionadas a esse nível educacional (Gráfico 2), e/ou à formação inicial ou continuada de 
professores, que, em nosso entendimento, é uma linha de pesquisa que merece atenção, uma vez que boa parte da publicação em BL está voltada aos alunos e seus resultados de aprendizagem.

Convém salientar que as questões de pesquisa apresentadas foram extraídas dos artigos completos, em periódicos com Fator de Impacto (FI) reconhecido pelo JCR/ISI, e cuja definição de BL compreende a integração intencional do ensino e aprendizagem presencial com o on-line (GRAHAM, 2006); (HORN; STAKER, 2015).

\section{Quadro 3 - Exemplos de questões de pesquisa em Blended Learning estruturados por tópicos}

\begin{tabular}{|c|c|}
\hline Tópico & Questões \\
\hline $\begin{array}{c}\text { Design } \\
\text { Instrucional }\end{array}$ & $\begin{array}{l}\text { Ao transformar um curso de Introdução à Patologia para mais de } 250 \text { estudantes de graduação, } \\
\text { demonstramos um modelo para o uso de } B L \text { em sessões de ensino em grandes grupos, que alcançaram } \\
\text { altos níveis de conclusão, satisfação e valor para o aprendizado. As reflexões dos alunos forneceram } \\
\text { evidências de que os vários componentes do curso foram usados sinergicamente em nosso modelo, } \\
\text { oferecendo uma experiência de aprendizado holística. (HERBERT et al., 2017). } \\
\text { Um modelo foi desenvolvido para ajudar os professores a usar uma abordagem blended, para projetar } \\
\text { intervenções de aprendizagem significativas para a fisiologia (em cursos de graduação). O objetivo do } \\
\text { modelo é incentivar o desenvolvimento metódico dessas intervenções de aprendizagem; enquanto o } \\
\text { propósito é fornecer ferramentas conceituais e de comunicação que possam ser usadas para visualizar, } \\
\text { dirigir e gerenciar processos para desenvolver ou selecionar intervenções de aprendizagem operacional, } \\
\text { apropriadas para o estudante do século XXI. (EAGLETON, 2017). } \\
\text { O autor apresenta um modelo que utiliza a avaliação por pares, para avaliar com mais precisão alunos } \\
\text { que participam de cursos em formato BL. O estudo visa responder as seguintes questões: Com que } \\
\text { precisão e eficiência é possível avaliar a participação individual do grupo no curso BL? Como avaliar } \\
\text { o impacto e a influência da avaliação por pares para os alunos que participam dos cursos de BL? O } \\
\text { uso de ferramentas } L M S \text { para projetar atividades de aprendizagem, nos cursos blended, incentiva os } \\
\text { alunos a trabalhar em grupos para resolver o problema ou não? (NGUYEN, 2017). }\end{array}$ \\
\hline $\begin{array}{l}\text { Resultado dos } \\
\text { Alunos }\end{array}$ & $\begin{array}{l}\text { O objetivo do artigo é triplo: (1) apresentar um modelo para prever a satisfação dos alunos com os } \\
\text { programas } B L,(2) \text { examinar o efeito da interação entre a experiência do instrutor e o sistema de gestão } \\
\text { de aprendizagem ( } L M S \text { ) na satisfação do estudante, em diferentes modos } B L \text {, e (3) investigar se } \\
\text { diferentes modos de } B L \text { exercerão influência sobre os objetivos percebidos e a satisfação dos alunos, } \\
\text { sua avaliação dos instrutores e a qualidade do } L M S \text {. (DIEP et al., 2017). }\end{array}$ \\
\hline Disposição & $\begin{array}{l}\text { Que tipo de perfil motivacional pode ser identificado entre os alunos de um programa de formação de } \\
\text { professores, em ambientes de aprendizagem on-line e blended (OBL)? Com base na teoria do valor das } \\
\text { expectativas, quais valores específicos estes alunos atribuem à sua participação na formação em OBL? } \\
\text { As implicações deste estudo mostram que: os alunos precisam de informações melhores e realísticas } \\
\text { sobre OBL. Um equilibrio ponderado entre momentos F2F e a distância é necessário. } \\
\text { (VANSLAMBROUCK et al., 2018). } \\
\text { O objetivo do estudo foi duplo: primeiro, explorar a relação entre os estilos de aprendizagem dos } \\
\text { alunos e sua participação on-line, em um curso de BL, e investigar as relações de participação on-line } \\
\text { dos alunos com o seu aprendizado e com a satisfação no curso. (CHENG; CHAU, 2016). }\end{array}$ \\
\hline Tecnologia & $\begin{array}{l}\text { Este artigo trata do uso de uma abordagem de métodos mistos (questionário, entrevista, observação e } \\
\text { revisão de documentos organizacionais), em uma investigação que buscou avaliar a infraestrutura } \\
\text { disponível de TIC, capaz de suportar o uso de tecnologias colaborativas da Web, em ambientes BL, } \\
\text { nas instituições de Ensino Superior da Tanzânia. (PIMA et al., 2016). }\end{array}$ \\
\hline Exploração & $\begin{array}{l}\text { Este estudo abordou o acesso dos alunos examinando as taxas de êxito e de abandono nos cursos } \\
\text { ofertados em BL, comparando-os às modalidades presencial e on-line [...]. Além disso, procurou avaliar } \\
\text { essas taxas considerando diferentes status de estudantes (minorias versus maioria). (DZIUBAN et al., } \\
\text { 2018). }\end{array}$ \\
\hline
\end{tabular}


(Conclusão)

\begin{tabular}{|c|c|}
\hline Comparação & $\begin{array}{l}\text { O objetivo foi avaliar o quão eficaz e eficiente é o e-learning e o BL, quando comparados com a } \\
\text { aprendizagem tradicional presencial, na Educação Ortodôntica. Também fornece uma comparação } \\
\text { entre o aprendizado presencial, o e-learning e o BL. (KUMAR, 2017). } \\
\text { Identificamos cinco subáreas de pesquisa e ilustramos usando estudos de caso: tecnologias } \\
\text { móveis, cursos on-line massivos abertos (MOOCs), bibliotecas digitais e projeto de objetos de } \\
\text { aprendizagem, aprendizado colaborativo, usando tecnologia digital, e treinamento de professores, } \\
\text { usando } B L \text {. (BORBA et al., 2016). }\end{array}$ \\
\hline $\begin{array}{l}\text { Desenvolvimento } \\
\text { Profissional }\end{array}$ & $\begin{array}{l}\text { O propósito deste estudo foi desenvolver um curso de formação de professores em serviço, do } \\
\text { Ensino Médio, no modelo } B L \text {, para aprendizagem da abordagem Hands-on, e examinar seus efeitos } \\
\text { sobre o conhecimento adquirido, autoeficácia e satisfação com a formação blended. (HO et al., } \\
\text { 2016). }\end{array}$ \\
\hline Demografia & $\begin{array}{l}\text { Observamos que as diferenças individuais desempenham um papel importante na aprendizagem dos } \\
\text { alunos e, portanto, este estudo considera que as características do aluno (como: idade, sexo, } \\
\text { autorregulação, atitudes, apoio familiar e social, bem como a gestão do volume de trabalho no BL) e o } \\
\text { histórico, em termos de aspectos demográficos e sociais, devem ser levados em consideração no design } \\
\text { de BL. (KINTU; ZHU, 2016). }\end{array}$ \\
\hline Interação & $\begin{array}{l}{[\ldots] \text { há mais que precisamos saber sobre como os alunos (de graduação em Ciência e Engenharia) }} \\
\text { interagem através de uma ferramenta de feedback corretivo imediato, em um ambiente de BL, e como } \\
\text { essas interações influenciam sua aprendizagem. Nossas descobertas mostram que certos padrões de } \\
\text { engajamento, com feedback, refletem estratégias de estudo produtivas e preveem, significativamente, } \\
\text { um desempenho mais alto. (CHEN; BRESLOW; DEBOER, 2018). }\end{array}$ \\
\hline Outros & $\begin{array}{l}\text { Que estratégias os instrutores apresentam para diferenciar os alunos, em um contexto de BL? Quais } \\
\text { são as crenças dos instrutores sobre o design de } B L \text { para abordar a diversidade dos alunos? Como } \\
\text { podem ser explicadas as diferenças, nas estratégias dos instrutores, para a instrução personalizada e, } \\
\text { nas crenças sobre o design de } B L \text {, para abordar a diversidade dos alunos? (BOELENS; VOET; DE } \\
\text { WEVER, 2018). }\end{array}$ \\
\hline
\end{tabular}

Fonte: Os autores (2019).

Ainda que o panorama de pesquisas com $B L$ seja amplo, com várias pesquisas voltadas ao Ensino Superior explorando o $B L$, em nível de curso, por iniciativas de professores que buscam inovar no ensino e na aprendizagem, poucos estudos oferecem orientação para adoção do $B L$ em nível institucional, bem como sobre como definir e medir o nível de utilização do BL (GRAHAM; WOODFIELD; HARRISON, 2013).

Nessa direção, encontramos os estudos de Graham, Woodfield e Harrison (2013) que forneceram uma estrutura institucional de adoção de $B L$, através da identificação de estratégias, estruturas e problemas específicos de suporte que as instituições normalmente tratam ao implementar o BL. E o estudo de Porter et al. (2014), no qual examinaram instituições de Ensino Superior dos Estados Unidos da América (EUA) que estavam em transição entre o primeiro e o segundo estágio de adoção do BL, de acordo com os estágios propostos em Graham, Woodfield e Harrison (2013).

\section{CONSIDERAÇÕES FINAIS}

Ao analisarmos as produções científicas de periódicos, compreendidas entre janeiro de 2015 e dezembro de 2018, visamos aprofundar o conhecimento sobre a temática do BL. Nesse sentido, buscamos identificar a maneira como o $B L$ vem desenvolvendo-se no cenário internacional, com um olhar sobre o nível de ensino ou contexto no qual se situam as pesquisas, os periódicos que podem ser considerados fontes primárias de estudo, os temas (ou tópicos) frequentes nas pesquisas com base no que elas exploram e, por fim, algumas questões de pesquisa abordadas nessas publicações.

De modo geral, nosso olhar sobre as publicações recentes indicou o crescimento, em nível internacional, da adoção e implementação do BL altamente direcionado à Educação Superior e 
conduzido, muito mais, por meio de iniciativas individuais e de grupo do que institucional. Acreditamos que isso se deve ao fato de o BL estar desenvolvendo-se a partir do EAD (E-Learning, em inglês, como é comumente citado) e, consequentemente, nas universidades, onde o ensino mediado pelas tecnologias digitais tem avançado, seja em processos de ensino semipresencial ou totalmente a distância.

Dessa forma, os diferentes artigos abordados evidenciam que as definições e discussões teóricas em torno da sua efetividade estão dando lugar às investigações que exploram a criação e implementação de modelos blended (EAGLETON, 2017) (NGUYEN, 2017). Descrições sobre o modo de configuração/implementação do BL (HERBERT et al., 2017) para um determinado assunto/conteúdo, em um curso/atividade, também são muito presentes nas pesquisas recentes. Do mesmo modo, estudos que revelam os resultados do BL com relação aos efeitos no aproveitamento, percepção e na satisfação dos alunos, conforme encontrado em Diep et al. (2017), são frequentes.

No universo das publicações exploradas, percebemos um movimento significativo de pesquisas dedicando-se: a redesenhar a sala de aula através de estratégias/práticas (NGUYEN, 2017); a verificar o quanto e de que modo a utilização do BL promove a aprendizagem de estudantes (DIEP et al. (2017); a demonstrar como os alunos o percebem e que expectativas são atendidas a partir desse modelo (VANSLAMBROUCK et al., 2018); a investigar como/quais TIC podem ser empregadas (PIMA et al., 2016); a compreender se/como a interação se transforma nos ambientes blended (CHEN; BRESLOW; DEBOER, 2018); e a determinar como os instrutores podem colaborar para o sucesso e efetivação da aprendizagem (BOELENS; VOET; DE WEVER, 2018).

Muitas das contribuições atuais, em termos de experiências de ensino e aprendizagem com $B L$, foram identificadas em programas educacionais de profissões da Saúde, mais precisamente 77 $(25,92 \%)$ dos artigos revisados. Isso pode ser evidenciado pelo periódico BMC Medical Education, que ocupou o $1^{\circ}$ lugar no ranking dos Top Ten, o que revela a flexibilidade do modelo para atender à demanda no ensino das diferentes áreas.

Dentre essas investigações, podemos destacar alguns exemplos: Dos Santos Czepula (2018), que analisou a implementação de uma metodologia ativa, em um modelo $B L$, nos processos de ensino e aprendizagem de estudantes de disciplinas de Farmácia; Wikander e Bouchoucha (2018), que apresentam uma visão geral do processo que leva à adequação bem-sucedida da Avaliação Clínica Estrutural Objetiva, para atender aos requisitos de um curso de Enfermagem, de pré-inscrição entregue através de BL; e Ratka-Krüger et al. (2018), que apresenta um estudo de caso das estruturas e resultados de um Programa de Desenvolvimento Profissional Contínuo (CPD), desenvolvido, através de BL, em Periodontologia.

O ensino de Inglês para Fins Específicos (IFE) é outra área que aproveita o potencial do $B L$ para enriquecer a aprendizagem. Alguns desses estudos são encontrados em: Pinto-Llorente (2017), em que os resultados mostram as percepções e atitudes dos alunos sobre a eficácia de ferramentas tecnológicas assíncronas, em atividades de $B L$, para melhorar sua competência gramatical em inglês como segunda língua; Banditvilai (2016), que apresentou um estudo de caso sobre o uso de BL para aprimorar as habilidades de linguagem e a autonomia dos alunos em aula de IFE, em um ambiente universitário asiático, na qual as estratégias de e-learning são usadas em paralelo com os métodos tradicionais. E Cañarte e Quevedo (2016), que buscaram viabilizar, através de análise e síntese, uma estratégia para o desenvolvimento da competência comunicativa oral, na modalidade $B L$, voltada à aprendizagem do inglês como língua estrangeira para alunos de Engenharia Civil.

Embora alguns periódicos se destaquem nas publicações relacionadas à temática, o número de títulos diferentes onde foram encontradas sugere que o $B L$, enquanto modelo, modalidade ou abordagem educacional, se propaga por inúmeras e diferentes áreas de conhecimento e programas, desde a graduação até a pós-graduação. Contudo, estão ligadas ao contexto da Educação.

O crescimento de pesquisas com $B L$, em diferentes países e continentes, revela a expansão da temática em cenários educacionais distintos. Em parte, isso acontece porque, nos modelos $B L$, mesmo sendo a tecnologia digital um dos seus componentes fundamentais para integração da aprendizagem presencial com a virtual, é possível promovê-lo (o modelo BL) a partir de diferentes infraestruturas tecnológicas. Desde que ela (tecnologia digital) comporte recursos, como celular, computador ou tablet; ou disponibilize ferramentas síncronas (Internet, AVA, MOOC); ou mesmo assíncronas (whatsapp e 
aplicativos de mensagens, e-mails, chats de texto), podemos pensar em combinar estratégias que potencializem o ensino e a aprendizagem mediados pelas tecnologias.

Uma das limitações desta investigação é que a RSL realizada compreendeu artigos publicados nas línguas inglesa, espanhola e portuguesa, essencialmente. A exploração de estudos similares, incorporando artigos em outras línguas, poderiam potencializar os resultados alcançados. Outra limitação, assim entendida, foi considerar a expressão "Blended Learning" apenas no título. Desse modo, um novo estudo poderia explorar diferentes combinações tanto no título quanto no assunto. Por fim, o volume de artigos a serem codificados dificultou uma exploração mais aprofundada, envolvendo a análise de aspectos metodológicos e dos dados produzidos.

Considerando que os professores são os principais tomadores de decisões pedagógicas em suas salas de aula (Graham; Robinson, 2007) e que "pouco se tem publicado sobre a adoção de BL pelo corpo docente" (KALETA; SKIBBA; JOOSTEN, 2007, p. 112), identificamos uma lacuna nas pesquisas relacionadas à formação docente em BL para integração das TIC ao ensino e à aprendizagem dos estudantes.

Como sugestões para trabalhos futuros, a pesquisa demonstra que o $B L$, com relação ao Desenvolvimento Profissional, se apresenta de modo discreto. Assim, entendemos que essa temática possa ser explorada, no que tange à formação inicial e continuada de professores, para investigar modelos de formação docente para a adoção e implementação do BL.

\section{REFERÊNCIAS}

BACICH, Lilian; NETO, Adolfo T.; DE MELLO TREVISANI, Fernando. Ensino híbrido: personalização e tecnologia na educação. Penso Editora, 2015.

BANDITVILAI, Choosri. Enhancing Student's Language Skills through Blended Learning. Electronic Journal of e-Learning, v. 14, n. 3, p. 220-229, 2016. Disponível em: http:/ / bit.ly/2mmew6Q. Acesso em: 7 dez. 2018.

BARDIN, Laurence. Análise de conteúdo. Lisboa: Edições 70, p. 225, 2010.

BOELENS, Ruth; VOET, Michiel; DE WEVER, Bram. The design of blended learning in response to student diversity in higher education: Instructors' views and use of differentiated instruction in blended learning. Computers \& Education, v. 120, p. 197-212, May. 2018. Disponível em: https://doi.org/10.1016/j.compedu.2018.02.009. Acesso em: 7 jan. 2019.

BORBA, Marcelo C., et al. Blended learning, e-learning and mobile learning in mathematics education. ZDM, v. 48, n. 5, p. 589-610, 2016. Disponível: https://doi.org/10.1007/s11858-016-0798-4. Acesso em: 7 dez. 2018.

CAÑARTE, Jaime Walter; QUEVEDO, Ned. Estrategia de aprendizaje combinado del inglés en las carreras técnicas. Transformación, v. 12, n.1, p. 41-51, 2016. Disponível em: http://bit.ly/2Nm0QEx. Acesso em: 5 dez. 2018.

CHEN, Xin; BRESLOW, Lori; DEBOER, Jennifer. Analyzing productive learning behaviors for students using immediate corrective feedback in a blended learning environment. Computers $\boldsymbol{\&}$ Education, v. 117, p. 59-74, fev. 2018. Disponível em: https://doi.org/10.1016/j.compedu.2017.09.013. Acesso em: 7 jan. 2019.

CHENG, Gary; CHAU, Juliana. Exploring the relationships between learning styles, online participation, learning achievement and course satisfaction: An empirical study of a blended learning course. British Journal of Educational Technology, v. 47, n. 2, p. 257-278, dez. 2016. Disponível em: https://doi.org/10.1111/bjet.12243. Acesso em: 5 dez. 2018. 
CHRISTENSEN, Clayton; M.; HORN, Michael. B.; STAKER, Heather. Ensino híbrido: uma inovação disruptiva? uma introdução à teoria dos híbridos. Maio 2013. 49 pág. Disponível em: https://www.christenseninstitute.org/publications/ensino-hibrido. Acesso em: 12 jan. 2016.

COSTA, Angelo Brandelli; ZOLTOWSKI, Ana Paula Couto. Como escrever um artigo de revisão sistemática. Manual de produção científica, p. 55-70, 2014.

DEPAEPE, Fien; VERSCHAFFEL, Lieven; KELCHTERMANS, Geert. Pedagogical content knowledge: A systematic review of the way in which the concept has pervaded mathematics educational research. Teaching and teacher education, v. 34, p. 12-25, 2013. Disponível em:

https://doi.org/10.1016/j.tate.2013.03.001. Acesso em: 6 jul. 2017.

DIEP, Anh-Nguyet, et al. Who or what contributes to student satisfaction in different blended learning modalities?. British Journal of Educational Technology, v.48, n.2. p. 473-489. 2017. Disponível em: https://doi.org/10.1111/bjet.12431. Acesso em: 5 dez. 2018.

DRYSDALE, Jeffery S., et al. An analysis of research trends in dissertations and theses studying blended learning. The Internet and Higher Education, v.17. p. 90-100, abril 2013. Disponível em: https://doi.org/10.1016/j.iheduc.2012.11.003. Acesso em: 21 mai. 2017.

DOS SANTOS CZEPULA, Alexandra Ingrid, et al. Active methodology and blended learning: An experience in pharmaceutical care. Currents in Pharmacy Teaching and Learning, v. 10, n.1, p. 106-111, jan./feb. 2018. Disponível em: https://doi.org/10.1016/j.cptl.2017.09.013. Acesso em: 15 ago. 2018.

DZIUBAN, Charles, et al. Blended learning: the new normal and emerging technologies. International Journal of Educational Technology in Higher Education, v.15, n.3, p. 1-16, feb. 2018. Disponível em: https://doi.org/10.1186/s41239-017-0087-5. Acesso em: 22 dez. 2018.

EAGLETON, Saramarie. Designing blended learning interventions for the 21st century student. Advances in physiology education, vol. 41, n. 2, p. 203-211, 2017. Disponível em: https://doi.org/10.1152/advan.00149.2016. Acesso em: 4 dez. 2018.

GARRISON, D. Randy; KANUKA, Heather. Blended learning: Uncovering its transformative potential in higher education. The internet and higher education, v. 7, n. 2, p. 95-105, 2004. Disponível em: http://www.anitacrawley.net/Resources/Articles/Garrison Kanuka2004.pdf. Acesso em: 15 ago. 2017.

GARRISON, D. Randy; VAUGHAN, Norman D. Blended learning in higher education: Framework, principles, and guidelines. John Wiley \& Sons, 2008.

GARRISON, D. Randy; VAUGHAN, Norman D. Institutional change and leadership associated with blended learning innovation: Two case studies. The internet and higher education, v. 18, p. 24-28, 2013. Disponível em: https://www.sciencedirect.com/ science/article/abs/pii/S1096751612000589. Acesso em: 13 ago. 2018.

GRAHAM, Charles R. Blended learning systems: definition, current trends, and future directions. In: BONK, C.J.; GRAHAM, C. R.; CROSS, J.; MOORE. M.J. (eds.) The handbook of blended learning: global perspectives, local designs. São Francisco: Pfeiffer Publishing, 2006. p. 30-41. Disponível em: http://www.publicationshare.com/graham_intro.pdf. Acesso em: 1 dez. 2016. 
GRAHAM, Charles R.; WOODFIELD, Wendy; HARRISON, J. Buckley. A framework for institutional adoption and implementation of blended learning in higher education. The internet and higher education, v. 18, p. 4-14, 2013. Disponível em: https://doi.org/10.1016/j.iheduc.2012.09.003. Acesso em: 13 ago. 2017.

HALVERSON, Lisa R., et al. An analysis of high impact scholarship and publication trends in blended learning. Distance Education, v. 33, n 3, p. 381-413, nov. 2012. Disponível em: https://doi.org/10.1080/01587919.2012.723166. Acesso em: 12 jul. 2018.

HALVERSON, Lisa R., et al. A thematic analysis of the most highly cited scholarship in the first decade of blended learning research. The Internet and Higher Education, v. 20, p. 20-34, jan. 2014. Disponível em: https://doi.org/10.1016/j.iheduc.2013.09.004. Acesso em: 15 ago. 2018.

HERBERT, Cristan, et al. A model for the use of blended learning in large group teaching sessions. BMC Medical Education, v. 17, n. 1, p. 197, nov. 2017. Disponível em: https://doi.org/10.1186/s12909-017-1057-2. Acesso em 15 dez. 2018.

HO, Vinh-Thang, et al. Blended learning model on hands-on approach for in-service secondary school teachers: Combination of E-learning and face-to-face discussion. Education and Information Technologies, v. 21, n. 1, p. 185-208, jan. 2016. Disponível em: https://doi.org/10.1007/s10639-0149315-y. Acesso em: 12 dez. 2018.

HORN, Michael B.; STAKER, Heather; CHRISTENSEN, Clayton. Blended: usando a inovação disruptiva para aprimorar a educação. Penso Editora, 2015.

KALETA, Robert; SKIBBA, Karen; JOOSTEN, Tanya. Discovering, designing, and delivering hybrid courses. Blended learning: Research perspectives, v. 111143, 2007.

KINTU, Mugenyi Justice; ZHU, Chang. Student Characteristics and Learning Outcomes in a Blended Learning Environment Intervention in a Ugandan University. Electronic Journal of e-Learning, v. 14, n. 3, p. 181-195, 2016. Disponível em: http://bit.ly/2I2qyuk. Acesso em: 12 dez. 2018.

KUMAR, Avinash. E-learning and blended learning in orthodontic education. APOS Trends in Orthodontics, v. 7, n.4, p. 188, jul. 2017. Disponível em: https://doi.org/10.4103/apos.apos_49_17. Acesso em: 12 dez. 2018.

MACDONALD, Janet. Blended learning and online tutoring: Planning learner support and activity design. Gower Publishing, Ltd., 2008.

MORAN, José. Educação híbrida: um conceito-chave para a educação, hoje. In: BACICH, L.; TANZI NETO, A.; TREVISANI, F. M. (Org.). Ensino híbrido: personalização e tecnologia na educação. Porto Alegre: Penso, 2015.

NGUYEN, Viet Anh. A peer assessment approach to project based blended learning course in a Vietnamese higher education. Education and Information Technologies, vol. 22, n. 5, p. 2141-2157, 2017. Disponível em: https://doi.org/10.1007/s10639-016-9539-0. Acesso em: 12 dez. 2018.

NORBERG, Anders; DZIUBAN, Charles D.; MOSKAL, Patsy D. A time-based blended learning model. On the Horizon, 2011. Disponível em: http://dx.doi.org/10.1108/107481211111. Acesso em: 12 dez. 2018. 
PIMA, John Marco, et al. Assessing the available ICT infrastructure for collaborative web technologies in a blended learning environment in Tanzania: A mixed methods research. International Journal of Education and Development using Information and Communication Technology, v. 12, n. 1, p. 37-52, 2016. Disponível em: http://bit.ly/2JRxHnh. Acesso em: 12 dez. 2018.

PINTO-LLORENTE, Ana $\mathrm{M}^{\mathrm{a}}$, et al. Students' perceptions and attitudes towards asynchronous technological tools in blended-learning training to improve grammatical competence in English as a second language. Computers in Human Behavior, v. 72, p. 632-643. Jul. 2017. Disponível em: https://doi.org/10.1016/j.chb.2016.05.071. Acesso em: 5 dez. 2018.

PORTER, Wendy W., et al. Blended learning in higher education: Institutional adoption and implementation. Computers \& Education, v. 75, p. 185-195, 2014. Disponível em: https://doi.org/10.1016/j.compedu.2014.02.011. Acesso em: 12 dez. 2018.

PORTER, Wendy W. et al. A qualitative analysis of institutional drivers and barriers to blended learning adoption in higher education. The internet and Higher education, v. 28, p. 17-27, 2016. Disponível em: https://www.sciencedirect.com/science/article/pii/S1096751615000469. Acesso em: 12 dez. 2018.

QSRINTERNATIONAL. What is NVIVO. Disponível em: https://www.qsrinternational.com/nvivo/what-is-nvivo. Acesso em: 2 jan. 2019.

RATKA-KRÜGER, Petra, et al. MasterOnline Periodontology and Implant Therapy-revisited after seven years: A case study of the structures and outcomes in a blended learning CPD. European Journal of Dental Education, v. 22, n.1, e7-e13. Dec. 2018. Disponível em: https://doi.org/10.1111/eje.12249. Acesso em: 15 jan. 2019.

ROZA, Jiani Cardoso da, et al. Aprendizagem na/da docência digital na perspectiva do BLearning e do TPACK na produção compartilhada de novas pedagogias. 2019. Tese de Doutorado. Universidade Federal de Santa Maria. Disponível em:

https://repositorio.ufsm.br/handle/1/19485. Acesso em: 2 set. 2019.

VANSLAMBROUCK, Silke, et al. Students' motivation and subjective task value of participating in online and blended learning environments. The Internet and Higher Education, v. 36, p. 33-40. Jan. 2018. Disponível em: https://doi.org/10.1016/j.iheduc.2017.09.002. Acesso em: 15 jan. 2019.

WIKANDER, Lolita; BOUCHOUCHA, Stéphane L. Facilitating peer-based learning through summative assessment - An adaptation of the Objective Structured Clinical Assessment tool for the blended learning environment. Nurse education in practice, v. 28, p. 40-45. Jan. 2018. Disponível em: https://doi.org/10.1016/j.nepr.2017.09.011. Acesso em: 15 jan. 2019.

Submetido: 02/05/2019

Aprovado: 13/10/2020 